\title{
AVALIAÇÃO DE CATALISADORES SOL-GEL E INDUSTRIAL FRENTE A REAÇÃO DE REFORMA A VAPOR DE METANOL
}

\author{
R. MENECHINI ${ }^{1}$, G. G. LENZI ${ }^{1}$, J. L. C. W. PIMENTA ${ }^{1}$, R. M. M. JORGE ${ }^{2}$, O. A. A. SANTOS ${ }^{1}$ e \\ L. M. M. JORGE ${ }^{1}$ \\ ${ }^{1}$ Universidade Estadual de Maringá, Departamento de Engenharia Química \\ ${ }^{2}$ Universidade Federal do Paraná, Departamento de Engenharia Química \\ E-mail para contato: menechini@yahoo.com.br,1mmj@deq.uem.br
}

\begin{abstract}
RESUMO - A reforma a vapor do metanol tem sido muito investigada para a produção de $\mathrm{H}_{2}$ com baixo teor de $\mathrm{CO}$ para células a combustível. Devido ao fato que diversas indústrias iniciaram a produção em larga escala de biometanol o qual tem se mostrado um excelente biocombustível. O biometanol não compete com a produção de alimentos, pois o mesmo pode ser produzido por diversas fontes como o resíduos de madeira e agrícolas, resíduos sólidos urbanos e uma série de outras matérias-primas. Por outro lado, a metodologia empregada na síntese do catalisador pode influenciar na atividade e seletividade do catalisador. O método de mistura química, sol-gel, geralmente produz catalisadores com desempenho superior aos catalisadores preparados pelos métodos clássicos. Neste trabalho foram comparadas a atividade e a seletividade de um catalisador comercial, HiFUEL R120, e um catalisador sol-gel de mesma composição, ambos aplicados na reforma a vapor de metanol.
\end{abstract}

\section{INTRODUÇÃO}

A reação de reforma a vapor de metanol vem sendo amplamente discutida e estudada mundo a fora. A reforma a vapor de metanol tem como principal componente do catalisador o cobre $(\mathrm{CuO})$, contudo uma melhor atividade e seletividade pode ser alcançada quando adicionados óxido de zinco $(\mathrm{ZnO})$ e alumina $\left(\mathrm{Al}_{2} \mathrm{O}_{3}\right)$ (JONES et al., 2008).

Devido ao fato dos principais catalisadores industriais para a reforma a vapor de metanol não utilizarem metais nobres, torna o metanol um excelente precursor para a obtenção do hidrogênio via reforma a vapor. É possível encontrar comercialmente diversos catalisadores para a reforma a vapor de metanol entre eles se encontra o HiFUEL R120, fabricado pela Alfa Aesar. Por mais que essa reação seja amplamente estuda, esse catalisador em especial não é frequentemente citado em trabalhos científicos (LO; WONG, 2011, 2012; KHZOUZ et al., 2012; MONTESANO; CHADWICK, 2012; CELIK et al., 2013).

Além dos metais utilizados na preparação do catalisador o método de preparação também exerce grande influência sobre o desempenho dos catalisadores, quando aplicados nos processos de reforma. Os catalisadores obtidos por meio do método da mistura química, também chamado método 


\section{9 a 22 de outubro de 2014 \\ Florianópolis/SC}

sol-gel, se apresentam mais ativos, seletivos, menor formação de coque e com melhor estabilidade térmica quando aplicados em muitas reações catalíticas heterogêneas (SARAGIOTTO COLPINI et al., 2008; GONÇALVES et al., 2013). Esse alto desempenho se deve ao fato destes catalisadores reunirem características importantes como alta porosidade, homogeneidade e área superficial elevada.

Diante do exposto a cima o presente trabalho teve como objetivo comparar o desempenho de um catalisador industrial e de um catalisador preparado pelo método sol-gel com a mesma composição, frente à reação de reforma a vapor do metanol.

\section{MATERIAIS E MÉTODOS}

A metodologia de preparação dos catalisadores sol-gel a base de cobre foi desenvolvida com base na rota de preparação de catalisadores sol-gel utilizadas por SANTOS (1999); GONÇALVES et al. (2005, 2006), LENZI et al. (2008) e (SARAGIOTTO COLPINI et al., 2008).

Nitrato de cobre tri hidratado $\left(\mathrm{Cu}\left(\mathrm{NO}_{3}\right)_{2} .3 \mathrm{H}_{2} \mathrm{O}\right)$, juntamente com nitrato de zinco hexa hidratado $\left(\mathrm{Zn}\left(\mathrm{NO}_{3}\right)_{2} \cdot 6 \mathrm{H}_{2} \mathrm{O}\right)$ foram dissolvidos em etanol anidro. Transferiu-se a solução etanólica dos sais precursores para um balão de três bocas $(500 \mathrm{~mL})$ acoplados a um agitador mecânico com pá de teflon e a um condensador de refluxo e mergulhado a um banho de glicerina ajustado a um sistema de aquecimento com temperatura controlada. Manteve-se a solução etanólica de $\mathrm{Cu} / \mathrm{Zn}$ sob agitação, por aproximadamente 15 minutos à temperatura ambiente. A esta solução, adicionou-se lentamente, por meio de um funil de adição, hexileno glicol, com leve aquecimento $\left(\approx 45^{\circ} \mathrm{C}\right)$; a quantidade de hexileno foi calculada com relação à massa do isopropóxido de alumínio usada, na razão mássica isopropóxido de alumínio/ hexileno glicol $=0,86$. Após a lenta adição de todo hexileno glicol elevouse a temperatura do banho para aproximadamente $95^{\circ} \mathrm{C}$ e manteve-se sob agitação nesta temperatura, durante 30 min, obtendo-se uma solução homogênea. Em seguida adicionou-se lentamente, com uma pequena espátula de vidro, sob agitação e temperatura ao redor de $90-95^{\circ} \mathrm{C}$, o isopropóxido de alumínio.

A solução permaneceu sob agitação à temperatura indicada durante 4 horas, na solução resultante adicionou-se outra solução (água deionizada e etanol). Finalmente adicionou-se uma terceira solução contendo água deionizada e etanol com o objetivo de completar a hidrólise. Nesta etapa, a solução formou um gel. Manteve-se o gel sob agitação durante 3 horas a $95^{\circ} \mathrm{C}$ para sua completa coagulação, e depois o balão de três bocas foi guardado em temperatura ambiente para envelhecimento por 85 horas, após esse período o gel é submetido a secagem e rota evaporador e a destilação à alto vácuo (GONÇALVES et al., 2005; SARAGIOTTO COLPINI et al., 2008). A última etapa de preparação do catalisador sol-gel foi a calcinação em mufla, a temperatura de calcinação foi determinada pela analise termogravimétrica.

A fim de avaliar os catalisadores industrial e o sol-gel sintetizado, ambos foram testados em um reator diferencial instalado no módulo de reforma a vapor indicado na Figura 1, o qual é constituído basicamente por cinco partes:

- alimentação de gases [B]; 
- alimentação de líquidos [A] e [C];

- separação dos condensáveis [F] e [G];

- forno com o reator $[\mathrm{E}]$;

- analise cromatográfica $[\mathrm{H}]$.

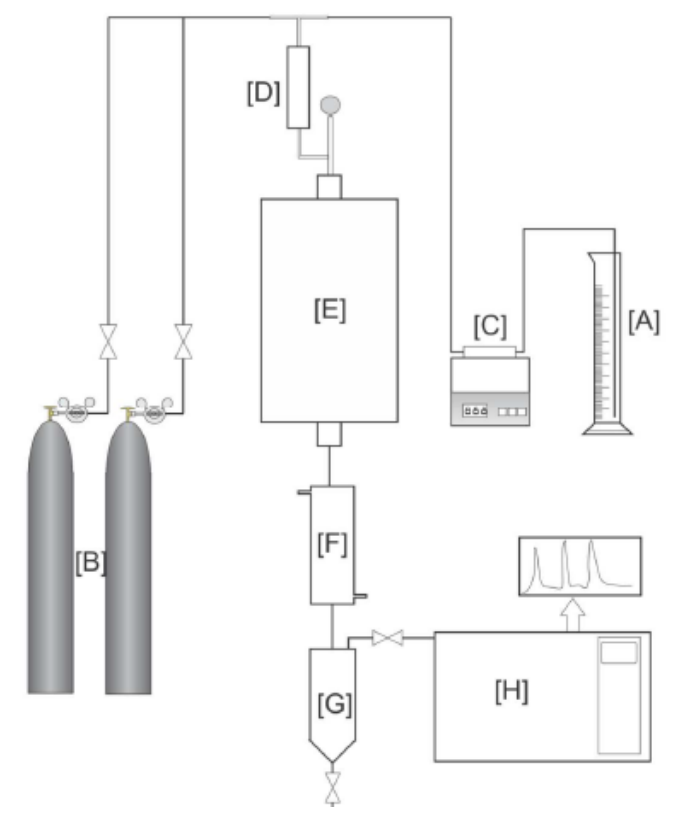

Figura 1 - Esquema do Módulo Experimental

O reator de $6,35 \mathrm{~mm}$ de diâmetro interno foi carregado com 500mg de catalisador HiFUEL R120 ou catalisador sol-gel de mesma composição mássica, após secagem e ativação in situ do catalisador, Tabela 1, realizou-se um ensaio de 12 horas e acompanhou-se o comportamento de desativação do catalisador, avaliando-se a conversão ao longo do tempo. Os parâmetros operacionais do teste são os estabelecidos na Tabela 2.

Tabela 1 - Rampa de ativação do catalisador.

\begin{tabular}{|c|c|c|}
\hline $\begin{array}{c}\text { Faixa de } \\
\text { Temperatura }\left({ }^{\circ} \mathrm{C}\right)\end{array}$ & Rampa $\left({ }^{\circ} \mathrm{C} / \mathrm{min}\right)$ & Patamar (min) \\
\hline $\mathrm{T}_{\mathrm{amb}}-200$ & 15 & 30 \\
\hline $200-300$ & 10 & 60 \\
\hline $300-400$ & 10 & 60 \\
\hline $400-500$ & 10 & 60 \\
\hline
\end{tabular}


Tabela 2 - Parâmetros Operacionais

\begin{tabular}{|c|c|}
\hline $\begin{array}{c}\text { Temperatura do pré- } \\
\text { aquecedor }\end{array}$ & $200^{\circ} \mathrm{C}$ \\
\hline $\begin{array}{c}\text { Vazão Mássica (solução de } \\
\text { metanol: água) }\end{array}$ & $1,0 \mathrm{~g} \cdot \mathrm{min}^{-1}$ \\
\hline Temperatura do reator & $300^{\circ} \mathrm{C}$ \\
\hline Temperatura de forno & $390^{\circ} \mathrm{C}$ \\
\hline Razões molares* & 3,0 \\
\hline
\end{tabular}

*A razão molar é dada pela razão de moles de água por moles de metanol

Os parâmetros utilizados para quantificar o desempenho do catalisador no reator diferencial são definidos como se segue nas equações 3 e 4.

Conversão de Metanol $(\%)=\frac{F_{\mathrm{CO}_{2}}+F_{\mathrm{CO}}}{F_{\mathrm{CH}_{3} \mathrm{OH}}} \cdot 100$

Seletividade $H_{2} / C O=\frac{C_{H_{2}}}{C_{C O}}$

onde $\mathrm{F}_{\mathrm{CO} 2}$ e $\mathrm{F}_{\mathrm{CO}}$ são vazões molares na saída do reator de dióxido de carbono e monóxido de carbono respectivamente, $\mathrm{F}_{\mathrm{CH} 3 \mathrm{OH}}$ é a vazão molar de entrada no reator de metanol, $\mathrm{C}_{\mathrm{H} 2}$ e $\mathrm{C}_{\mathrm{CO}}$ são as concentrações molares de hidrogênio e monóxido de carbono respectivamente.

\section{RESULTADOS E DISCUSSÕES}

A fim de determinar a melhor condição de calcinação do catalisador sol-gel foi realizado uma análise termogravimétrica, Figura 2, essa análise indicou que o catalisador apresenta apenas uma região de perda de massa significante. Esse região compreende a temperatura de $220^{\circ} \mathrm{C}$ a $350^{\circ} \mathrm{C}$ e corresponde à aproximadamente $35 \%$ da perda de massa do catalisador, essa perda de massa é devido a decomposição dos nitratos, evaporação e carbonização dos solventes residuais.

Os resultados indicaram que para as mesmas condições de operação o catalisador sol-gel se mostrou muito superior ao catalisador industrial, Figura 1. O catalisador sol-gel sintetizado apresentou uma desativação lenta em relação ao catalisador industrial, sendo possível constatar isso quando tomamos os coeficientes angulares das retas de ajuste dos dados, que apresentam coeficientes iguais a $0,028 \mathrm{~h}^{-1}$ e $0,070 \mathrm{~h}^{-1}$, respectivamente para catalisador sol-gel e industrial. 
Os resultados preliminares de conversão e estabilidade foram promissores para o catalisador sol-gel, apresentando conversão média de 33\% enquanto que o catalisador industrial apresentou conversão média de $8 \%$, quatro vezes inferior à conversão do sol-gel. A atividade apresentada pelo catalisador sol-gel é interessante pois é um catalisador ideal para compor micro reatores a fim de serem utilizados em sistemas portáteis de geração de energia elétrica baseados em células a combustíveis, a exemplo sistemas para alimentar notebook, celulares, tablets, entre outros.

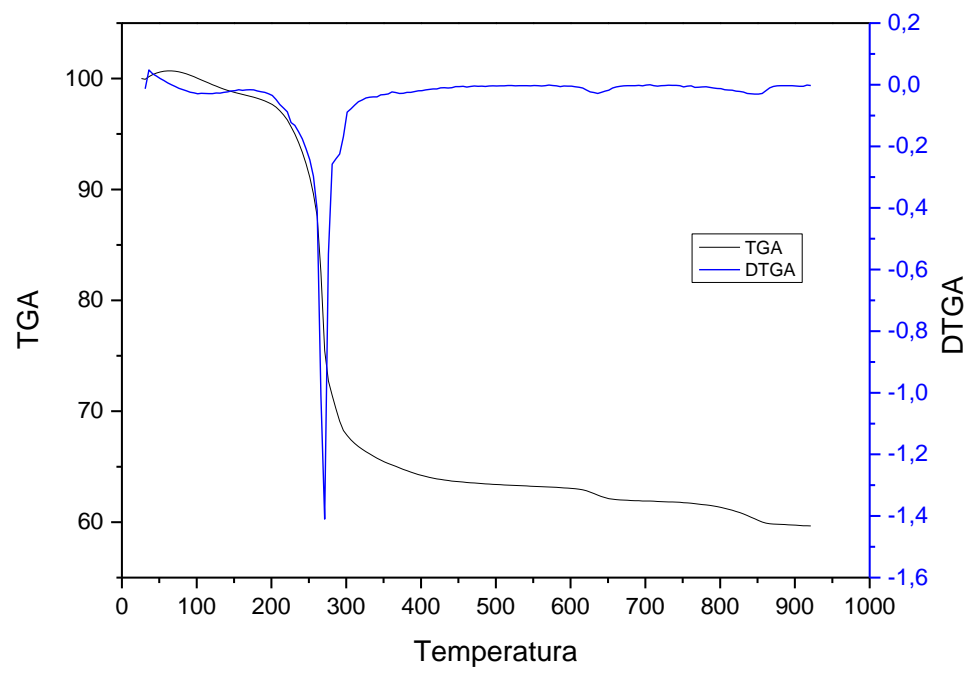

Figura 2 - Perfil de ATG/DTGA para o catalisador sol-gel

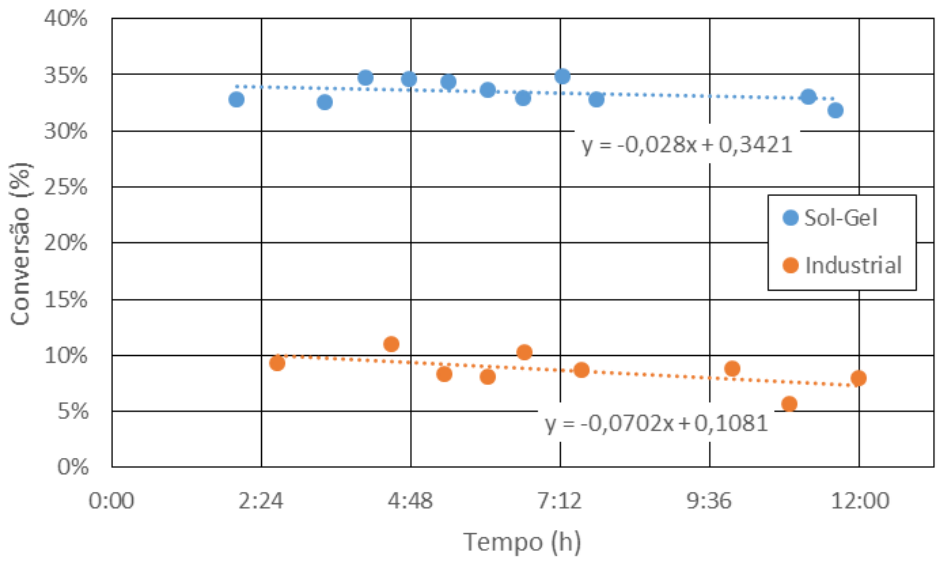

Figura 3 - Conversão do Catalisador Industrial e Sol-Gel 


\section{CONCLUSÕES}

Os testes reacionais em reator diferencial para os catalisadores sol-gel e industrial mostraram que a conversão do catalisador sol-gel de mesma composição do industrial é 4 vezes maior para as mesmas condições operacionais.

O catalisador sol-gel produziu uma corrente de $200 \mathrm{~mL}$ min de hidrogênio o que é suficiente para abastecer uma célula a combustível de 20 watts, isso é possível utilizando apenas $500 \mathrm{mg}$ de catalisador sol-gel a base de cobre.

O catalisador sol-gel se mostra promissor, sendo necessário um estudo mais aprofundado de caracterização da estrutura do mesmo a fim de esclarecer os motivos do aumento de desempenho do catalisador sol-gel em relação ao catalisador industrial sendo que ambos os catalisadores possuem a mesma composição.

\section{REFERÊNCIAS}

CELIK, G.; ARINAN, A.; BAYAT, A.; et al. Performance of Silicotungstic Acid Incorporated Mesoporous Catalyst in Direct Synthesis of Dimethyl Ether from Syngas in the Presence and Absence of CO 2. Top Catal, v. 56, p. 1764-1774, 2013.

ENDO, F. Investigação da Reforma do Metanol em Reator de Leito Fixo, 2009. Universidade Estadual de Maringá.

GONÇALVES, G.; COLPINI, L. M. S.; MENECHINI NETO, R.; SANTOS, O. A. A.; JORGE, L. M. M.; LENZI, M. K.; Coke formation quantitative analysis on impregnated and sol-gel catalysts applied the dry reform of methane. International Journal of Materials Engineering and Technology, v. 10, n. 1, p. 45-65, 2013.

GONÇALVES, G.; MARTINELLI, F. Q.; MAIERU, C.; et al. Catalisadores sol-gel de Ni-SiO2 e Ni-Al2O3 aplicados na reforma de metano com CO2. Acta Scientiarum. Technology, v. 27, n. 1, p. 17-22, 2005.

JONES, S. D.; NEAL, L. M.; HAGELIN-WEAVER, H. E. Steam reforming of methanol using $\mathrm{Cu}-\mathrm{ZnO}$ catalysts supported on nanoparticle alumina. Applied Catalysis B: Environmental, v. 84, n. 3-4, p. 631-642, 2008.

KHZOUZ, M.; WOOD, J.; POLLET, B.; BUJALSKI, W. Characterization and activity test of commercial $\mathrm{Ni} / \mathrm{Al} 2 \mathrm{O} 3, \mathrm{Cu} / \mathrm{ZnO} / \mathrm{Al} 2 \mathrm{O} 3$ and prepared $\mathrm{Ni} \mathrm{e} \mathrm{Cu} / \mathrm{Al} 2 \mathrm{O} 3$ catalysts for 
hydrogen production from methane and methanol fuels. International Journal of Hydrogen Energy, v. 38, n. 3, p. 1664-1675, 2012.

LO, K.; WONG, S. A passively-fed methanol steam reformer with catalytic combustor heater. International Journal of Hydrogen Energy, v. 36, n. 17, p. 10719-10726, 2011.

LO, K.-F.; WONG, S.-C. A passively-fed methanol steam reformer heated with two-stage bifueled catalytic combustor. Journal of Power Sources, v. 213, p. 112-118, 2012.

MONTESANO, R.; CHADWICK, D. Combined methanol and dimethyl ether synthesis from $\mathrm{CO} / \mathrm{H} 2$ : Phosphorus mediated deactivation. Catalysis Communications, v. 29, p. 137-140, 2012.

SARAGIOTTO COLPINI, L. M.; LENZI, G. G.; MACEDO COSTA, C. M. Determination of surface sites using infrared spectroscopy for mixed oxides of vanadium obtained by the sol-gel method. Journal of Non-Crystalline Solids, v. 354, n. 42-44, p. 4816-4822, 2008. 